\title{
Erratum: Bounds on the speed of sound in dense matter, and neutron star structure [Phys. Rev. C 95, 045801 (2017)]
}

\author{
Ch. C. Moustakidis, T. Gaitanos, Ch. Margaritis, and G. A. Lalazissis
}

(Received 4 May 2017; published 26 May 2017)

DOI: 10.1103/PhysRevC.95.059904

We have noticed three typographical errors in the original paper. In the third line on the right-hand side of Eq. (3), $\mathcal{E}_{c}$ should be replaced by $\mathcal{E}_{0}$. Now, the correct Eq. (3) should read

$$
P(\mathcal{E})= \begin{cases}P_{\text {crust }}(\mathcal{E}), & \mathcal{E} \leqslant \mathcal{E}_{\text {c-edge }}, \\ P_{\mathrm{NM}}(\mathcal{E}), & \mathcal{E}_{\text {c-edge }} \leqslant \mathcal{E} \leqslant \mathcal{E}_{0}, \\ \left(\frac{v_{S}}{c}\right)^{2}\left(\mathcal{E}-\mathcal{E}_{0}\right)+P_{\mathrm{NM}}\left(\mathcal{E}_{0}\right), & \mathcal{E}_{0} \leqslant \mathcal{E} .\end{cases}
$$

On the right-hand side of Eq. (20), $\lambda$ must be replaced by $-\lambda$. Now, the correct Eq. (20) should read

$$
Q_{i j}=-k_{2} \frac{2 R^{5}}{3 G} E_{i j} \equiv-\lambda E_{i j} .
$$

In Fig. 4 the label $n / n_{0}$ for the horizontal axis must be replaced by $n_{0} / n_{s}$.

The errors were in the presentation and not in the calculations, so the results (including the figures and conclusions) are not changed. 